Document downloaded from:

http://hdl.handle.net/10251/80759

This paper must be cited as:

Pastor Soriano, JV.; García Oliver, JM.; López, JJ.; Mico Reche, C. (2016). Application of UV Visible Light Absorption and Scattering technique to low absorption fuels under diesellike conditions. Fuel. 179:258-266. doi:10.1016/j.fuel.2016.03.080.

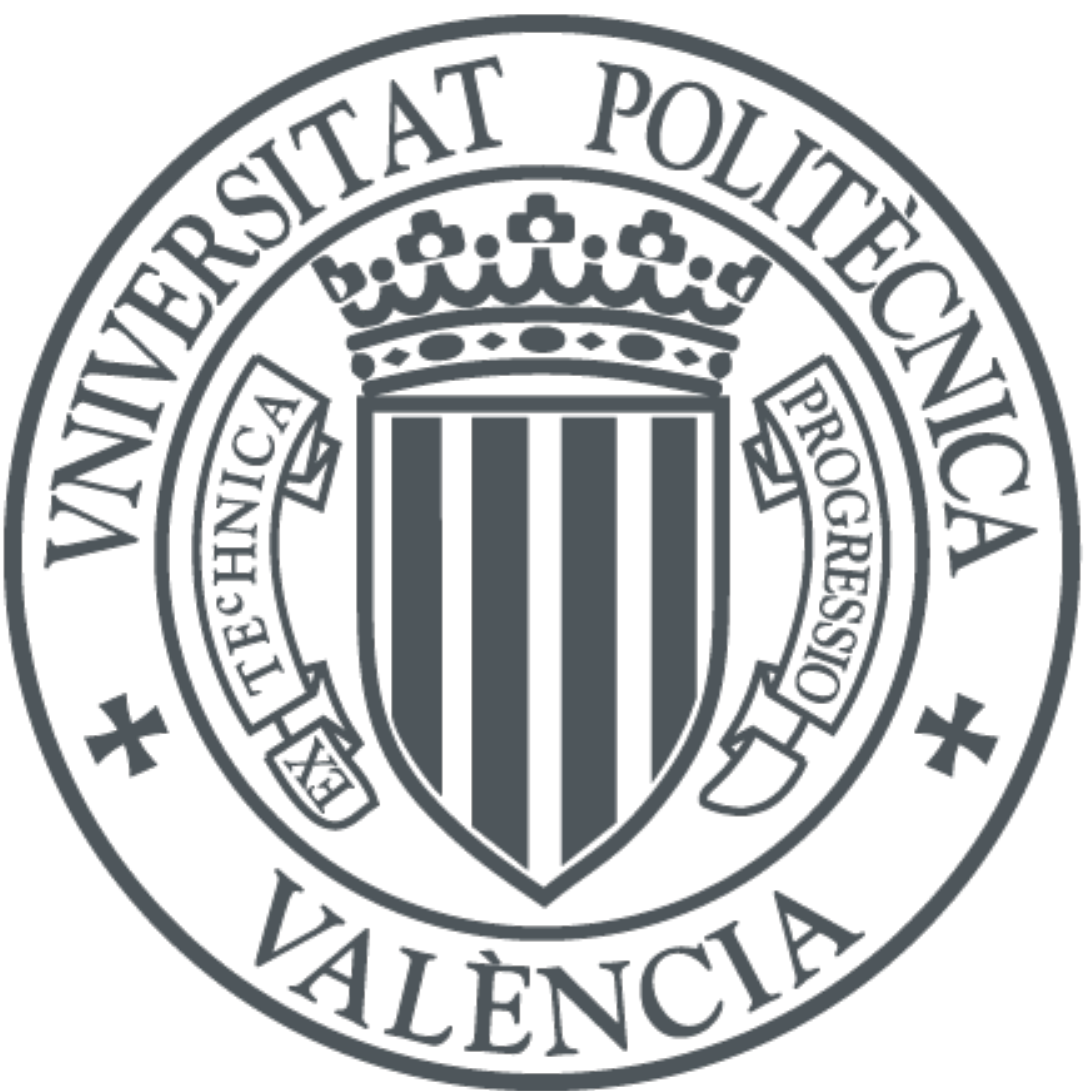

The final publication is available at

http://dx.doi.org/10.1016/j.fuel.2016.03.080

Copyright Elsevier

Additional Information 


\title{
Application of UV-Visible Light Absorption and Scattering technique to low absorption fuels under diesel-like conditions
}

\author{
J.V. Pastor ${ }^{\mathrm{a}, *}$, J.M. García-Oliver ${ }^{\mathrm{a}}$, J.J. López ${ }^{\mathrm{a}}$, C. Micó ${ }^{\mathrm{a}}$ \\ ${ }^{a}$ CMT-Motores Térmicos, Universitat Politècnica de València, Camino de Vera s/n, 46022 \\ Valencia, Spain
}

\begin{abstract}
Light Absorption and Scattering technique (LAS) has been applied for the measurement of fuel vapor distribution in diesel-type sprays. This technique is usually limited to fuels with relatively high absorptivity, which are sometimes not commonly used as surrogate fuels. In the present paper, a comparison of fuels with very different absorptive properties has been made to determine the range of application of the methodology. A calibration procedure has been applied to n-decane (DEC), a binary blend of n-decane and n-hexadecane (50DEC) and three blends of n-heptane with a highly-absorpting fuel (HEPB1, HEPB2 and HEPB3). This methodology enables the in-situ quantification of absorption coefficients at high pressure and temperature by creating a uniform mixture inside the cylinder. Results have been later applied for the quantification of fuel vapor distribution in sprays for DEC, 50DEC and HEPB3. Results obtained with these range of fuels have enabled to establish the limit in terms of absorption coefficient needed to get consistent results with the technique.

Keywords: UV-VIS Light Absorption and Scattering, direct injection, fuel, mixture formation, n-alkanes
\end{abstract}

\footnotetext{
*Corresponding author

Email address: jpastor@mot.upv.es (J.V. Pastor)
} 
1 1. Nomenclature

$\begin{array}{ll}\text { LAS } & \text { Light Absorption and Scattering } \\ \text { UV } & \text { Ultraviolet light } \\ \text { VIS } & \text { Visible light } \\ \text { TDC } & \text { Top Dead Centre } \\ \text { DEC } & \text { n-Decane } \\ 50 \mathrm{DEC} & \text { 50\%n-Decane/50\%n-Hexadecane } \\ \text { HEP } & \text { n-Heptane } \\ \text { HAF } & \text { multi-component high absorption fuel } \\ \text { HEPB\# } & \text { mixture of HEP and HAF } \\ \lambda & \text { Wavelength } \\ M W & \text { Molecular weight } \\ L & \text { Optical path } \\ I_{0} & \text { Reference light intensity } \\ I & \text { Attenuated light intensity } \\ \varepsilon & \text { Absorption coefficient } \\ \rho_{v f} & \text { vapor fuel partial density } \\ Y_{f} & \text { vapor fuel mass fraction } \\ \text { LoS } & \text { Line-of-sight } \\ \text { R } & \text { Ratio of droplet optical thickness at } 280 \text { and } 560 \mathrm{~nm} \\ \phi_{e q} & \text { Equivalent diameter } \\ d_{0} & \text { Nozzle exit diameter } \\ \rho_{f} & \text { Fuel density at injection conditions } \\ \rho_{a} & \text { Ambient gas density } \\ \text { NO } & \text { Nominal thermodynamic conditions } \\ \text { LD } & \text { Low density thermodynamic conditions } \\ \text { HT } & \text { Hight temperature thermodynamic conditions } \\ & \end{array}$

\section{2. Introduction}

$4 \quad$ Many efforts on internal combustion engine research are focused on reducing 5 pollutant formation. The more and more restrictive regulations force the devel- 
6 opment of new techniques and technologies, while improving the current ones.

7 One of the main research topics in this regard is the study of the evaporation of fuel and subsequent mixing with air. Especially the latter process has been proved to have a strong impact on combustion and pollutant formation in the spray [1. Over the past decades, many experimental diagnostic methods have been developed in order to characterize quantitatively the fuel distribution. Raman Spectroscopy allows the measurement of local fuel/air ratio [2]. However, the low signal strength limits measurement to a reduced area and requires careful signal-to-noise ratio considerations. In contrast, Planar Rayleigh Scattering (PRS) and Planar Laser Induced Fluorescence (PLIF) present more intense signals and allow spatially resolved measurements. On the one hand, PRS can be only applied in total absence of liquid droplets, which in practice means starting measurements further downstream of the stabilized liquid length 2 , 3, 4, On the other hand, PLIF has been widely employed to determine both vapor and liquid phase concentrations simultaneously (Exciplex PLIF) [5, 6, 7, 8. Nevertheless, difficulties are usually found due to quenching with other molecules or cross-talk between the monomer (vapor) and the exciplex (liquid) fluorescences. Besides, quantitative measurements under high temperature become difficult due to a strong dependence of fluorescence on this parameter [6].

Light Absorption and Scattering (LAS) technique is based on the fact that the phenomena governing light interaction with fuel can be either absorption or scattering, depending on the light spectrum and the size range of the fuel particles (i.e. droplets or molecules) relative to wavelength. Mancaruso and Vaglieco 9] showed extinction spectra of diesel fuel within an optical engine. Their results evidence a strong absorption in the UV, mainly due to the presence of aromatic molecules, while the spectra in the visible range is flat, which is due to liquid scattering. If absorption signal is isolated, fuel concentration can be obtained by means of Lambert-Beer's law. The first applications of LAS were based on the combination of infrared and visible wavelengths. However, infrared extinction usually presents strong temperature dependence and it can be interfered by the absorption of water vapor or heat radiation from hot sur- 
faces. Based on the same principle, the Ultraviolet-Visible Light Absorption and Scattering (UV-VIS LAS) was developed by Suzuki 10, and improved by Zhang [1] for application under high pressure and high temperature conditions. UV-VIS LAS is not influenced by water vapor or heat radiation, and temperature dependence is weaker than in other techniques. Besides, as both wavelengths are relatively close, several simplifications can be applied without affecting measurement accuracy. Therefore, UV-VIS LAS technique is regarded as a promising tool for quantitative measurement of concentration distribution for fuel sprays, in presence of both vapor and liquid.

One of the main requirements for the application of UV-VIS LAS is that the fuel under study has to be absorbent in the near UV range (between 250 and $300 \mathrm{~nm}$ ) while being transparent for the visible wavelengths. The absorption spectrum strongly depends on the fuel molecule itself. Most of the implementations available in the literature use complex fuels with high UV absorptivity [10, 11, 12, 13, 14, 15]. With the aim of expanding the range of application of UV-VIS LAS, this work addresses the application of this technique to measure fuel vapor distribution of two n-alkanes under diesel-like conditions. These type of fuels have been commonly used as surrogates of more complex ones. However, they present low absorption in the near-UV range. In current work, n-Decane and a $50 \%$ mass blend of n-Decane and n-Hexadecane have been investigated. In parallel, some more absorptive fuel blends have also been evaluated and compared with the other two to analyse the validity of results obtained. In addition, a calibration methodology for in-situ measurements of the absorption coefficient of each fuel is presented and validated.

\section{Experimental methodology}

\subsection{Experimental facility}

Tests have been performed in an optically accessible single cylinder engine. A detailed description can be found at [16]. The facility is based on a 2-stroke single cylinder engine (Jenbach JW 50), with 3 liter displacement. The engine is 
motored at low speed (500 rpm) and the intake and exhaust processes are handled by transfers on the liner. A schematic of the engine is depicted in figure 1 (left). The facility has been operated under non-reactive conditions in a closed loop mode, where in-cylinder air is fully replaced by nitrogen. When the exhaust gases leave the cylinder, they flow through an intercooler and a cyclonic filter to remove the rests of fuel and oil. This ensures proper operating conditions for a roots compressor, which is used to assist the engine charge management. In-cylinder thermodynamic conditions are controlled by the intake air temperature and pressure. The first one is regulated by two sets of electrical resistors. Between them, the circuit is refilled with nitrogen through an electronic valve to achieve the desired intake pressure, compensating blow-by and leak losses. The engine is operated under skip-fired mode, so that in-cylinder conditions are not influenced by the remaining residual gases from previous combustion/injection cycles and temperature transients are avoided. Hence, an injection takes place every 30 cycles.

The cylinder head is specially designed to provide optical access to the combustion chamber, which was designed with a cylindrical shape in order to avoid wall impingement. The effective compression ratio is 15.7 . The chamber presents an upper port, where the injector is located, and four lateral accesses. A pressure transducer is installed in one of them, whereas the other three are equipped with oval-shaped quartz windows (88 $\mathrm{mm}$ long, $37 \mathrm{~mm}$ large and 28 mm thick). The cylinder head and engine temperature are controlled by a coolant recirculation system. Temperature was set to $353 \mathrm{~K}$, to guarantee good lubricity.

A common-rail injection system was used, with a single-hole piezoelectric injector. The orifice had conical shape (Ks factor equal to 1.5), with an outlet diameter of $140 \mu \mathrm{m}$ and $1 \mathrm{~mm}$ length. The injected mass is so low that thermodynamic conditions inside the combustion chamber are barely affected by the fuel evaporation. Temperature of the injector holder cooling was the same as for the cylinder head. Hence, due to the low injection frequency, the injected fuel temperature can be considered the same. 


\subsection{Operating conditions}

An experimental matrix has been designed, which includes variations of both in-cylinder pressure and temperature. A nominal point has been defined (NO), together with lower density (LD) and higher temperature (HT) points. Compared to NO, LD is obtained by lowering intake pressure at constant temperature, while the HT is obtained by increasing intake temperature at constant pressure. Conditions inside the cylinder have been calculated from measured in-cylinder pressure, using a first-law thermodynamic analysis. A similar procedure has been followed in [16, 17, where a detailed explanation can be found. It takes into account blow-by, heat losses and mechanical deformations. The trapped mass is estimated using the intake temperature and volume at the exhaust vent closure. Therefore, temperature along the engine cycle can be calculated using the equation of state and correcting the air trapped mass with blow-by estimations. Air mass and density are also required for the absorption calibration methodology, as described in the upcoming sections. In-cylinder pressure trace and the derived gas density for the three operating points is presented in figure 1(right). The injection pressure was set at $100 \mathrm{MPa}$ for all the cases.

The vapor fuel concentration has been measured for n-Decane (DEC) and a $50 \%$ blend in mass of n-Decane and n-Hexadecane (50DEC). A more absorptive fuel has been also employed, which was obtained by diluting a highly absorption blend of diferent single-component fuels (HAF), and pure n-Heptane (HEP), which has a negligible absorption coefficient. All fuels were purchased with a 95\% purity. Different blending dilutions have been considered to span a range of absorption coefficient values of the blend. A summary of the composition of the different fuels is summarized in table 1 .

\section{UV-VIS LAS Methodology}

When light is transmitted through a mixture of vapor and droplets, it is attenuated according to the Bouguer-Lambert-Beer law as follows: 


$$
\ln \left(\frac{I_{0}}{I}\right)=\int_{0}^{L} \frac{1}{M W} \varepsilon(\lambda) \rho_{v f} 100 d x+\int_{0}^{L} Q_{e x t}(\lambda) d x
$$

where $\lambda$ is wavelength, $\varepsilon$ is the absorption coefficient of fuel vapor $\left(l \cdot \mathrm{mol}^{-1}\right.$. $\left.\mathrm{cm}^{-1}\right), \rho_{v f}$ is the vapor fuel partial density $\left(\mathrm{kg} / \mathrm{m}^{3}\right), M W$ is the molecular weight of fuel $(\mathrm{g} / \mathrm{mol}), L$ is the optical path length $(\mathrm{m})$, and $Q_{e x t}$ is the extinction coefficient of a cloud of droplets. The first term on the right side of equation 1 corresponds to light attenuation due to absorption by vapor molecules, while the second term is the extinction due to droplets, which includes scattering and absorption losses.

UV-VIS LAS is based on the combination of attenuation measurements at two wavelengths, the first one in the ultraviolet (UV) range and the other in the visible (VIS) range. In this work, 280 and $560 \mathrm{~nm}$ were chosen. Two main hypotheses are assumed:

- Fuel molecules will not absorb light in the visible range neither in the form of droplets nor in vapor phase.

- UV absorption by fuel droplets is negligible compared to scattering.

Suzuki et al. [10] evaluated the drop optical thickness at $280 \mathrm{~nm}$ and $560 \mathrm{~nm}$ for $\alpha$-dimethylnaphtalene and concluded that the hypothesis of non-absorbance from liquid droplets can be applied. However, close to the nozzle region a certain error can affect the measurement since the droplet number density is too high. This error is minimized if vapor optical thickness dominates the total extinction. If both 280 and 560 signals are combined, the following expression can be derived from equation 1

$$
\overline{\rho_{v f}}=\frac{M W}{100 \cdot \varepsilon\left(\lambda_{U V}\right)}\left[\ln \left(\frac{I_{0}}{I}\right)_{U V}-R \ln \left(\frac{I_{0}}{I}\right)_{V I S}\right]
$$

where $\overline{\rho_{v f}}$ is the average vapor partial density along the optical path, as LAS technique is based on Line-of-Sight (LoS) measurements. The $R$ term is the ratio of the drop optical thickness at the two wavelengths. From now on, the term within brackets will be referred to as absorption. Billings et al. [18] examined 
the variation in drop optical thickness for their application at $3390 \mathrm{~nm}$ and 632 nm. Calculations conducted for the present work show similar results in the UV-VIS range [19, 20]. It was observed that $\mathrm{R}$ varies mainly with the droplet diameter (for two fixed wavelengths). Below $25 \mathrm{um}, \mathrm{R}$ varies between 0.9 and 1 while for droplets larger than this size $\mathrm{R}$ is almost 1 . For the present work a range between 20 and 60 um was considered and an average value of $\mathrm{R}=0.976$ was used.

The optical set-up is presented in figure 2, A continuous broadband $1000 \mathrm{~W}$ $\mathrm{Hg}$ (Xenon) Arc lamp was utilized, in combination with a diaphragm and a diffuser to create a uniform point light source. This lamp is characterized by a continuous emission spectra from 250 to $2400 \mathrm{~nm}$, with enough intensity to replace the commonly used Nd:YAG pulsed laser [11, 12, 13, 14, 15]. A parabolic mirror of $150 \mathrm{~mm}$ diameter was employed to create a collimated light beam through the combustion chamber. The light beam is collected at the other side of the engine by a $75-\mathrm{mm}$ diameter quartz lens, which focalizes light on both detectors. A square quartz beam splitter (50 mm side) was positioned just after the lens, to divide light in two different beams (50\% transmitted and $50 \%$ reflected intensity in the whole working range). For both UV and VIS wavelengths, a digital ICCD image system Andor iStar was utilized, with a $50 \mu s$ exposure time. Light was filtered just prior to the detectors by two interference filters centred at 280 and $560 \mathrm{~nm}$ respectively (10 nm FWHM).

Simultaneously to LAS measurements, MIE scattering images from the liquid droplets were registered to identify the maximum liquid length. For that purpose, a third camera (ICCD LaVision Dynamight) was utilized, due to the low intensity of the collimated light beam. The procedure followed to register and process the signal is described in [21.

\subsection{Absorption coefficient calibration}

According to the literature, the absorption coefficient can be strongly affected by thermodynamic conditions [12, 22, Moreover, significant differences have been reported among different fuels. For this reason, it becomes necessary 
to characterize the absorptivity of each fuel under engine operating conditions. A methodology is proposed in the current work, based on creating a homogeneous mixture inside the cylinder with known concentration, temperature and pressure. Thus, if light absorption is measured under this conditions, it is possible to apply equation 2 to obtain the absorption coefficient at known thermodynamic conditions.

Trapped air mass and in-cylinder density were derived from the pressure signal, while the amount of fuel injected was previously measured for all the fuels, as described in $\left[23,24\right.$. Then, the average fuel mass fraction $\left(\overline{Y_{f}}\right)$ inside the cylinder can be calculated and hence $\overline{\rho_{v f}}=\overline{Y_{f}} \cdot \rho_{c}$. In order to achieve the homogeneous mixture, fuel was injected early in the cycle, just after the transfer closing (at -80.5 CAD). Due to the large displacement of the engine, long energizing times and high injection pressures were required to introduce enough mass of fuel to obtain a measurable concentration.

Caracterization was performed for the different blends at the operating conditions summarized in table 2. For each test conditions, 50 images of the light beam with fuel $(I)$ and without fuel $\left(I_{0}\right)$ were registered alternatively. Each set of images was averaged and by comparing to the vapor concentration, the absorption coefficient was calculated. For most of the cases, the procedure was repeated at different crankangle positions after TDC, which made it possible to calculate $\varepsilon$ for different combinations of pressure and temperature caused by piston motion. Moreover, measurements at different in-cylinder conditions but at the same crankangle positions enabled the comparison of points with the same pressure but different temperature or vice-versa.

Finally, the absorption spectrum and the absorption coefficient at $280 \mathrm{~nm}$ were measured at standard temperature and pressure (STP), for all the fuels included in the calibration process. The same light source as the one described previously was used, while absorption was measured with a UV-VIS spectrometer AvaSpec $2048 \mathrm{~L}$ and quartz sample cell of $5 \mathrm{~mm}$. 


\subsection{Spray measurements}

For measurements of fuel spatial distribution within sprays under engine conditions, a long energizing time was set so the spray was stabilized before the injection finished. The injector was triggered at $6 \mathrm{CAD}$ before TDC, while the actual injection started approximately at 5 CAD before TDC. The energizing time of the injector was set to $2000 \mu \mathrm{s}$ (6 CAD) resulting on a total injection duration of around $5000 \mu \mathrm{s}(15 \mathrm{CAD})$ due to the hydraulic delay. Images were taken at -3 CAD (1000 $\mu$ s after injector was triggered) before TDC.

The reduced size of the neutral density filter limited the field of view, so all the receiving optics were spatially shifted to measure the whole spray, with a precision translational stage. Light was registered at three positions along the spray axis. The effective length of the field of view was $45 \mathrm{~mm}$, while the optics were displaced $25 \mathrm{~mm}$ between two consecutive positions. Thus, an overlap of $20 \mathrm{~mm}$ was ensured, which was the base to merge the three images into a single one.

For each test condition and measuring position, 50 images were registered. Each set of images was averaged, merged and finally the attenuation was calculated at each wavelength. The VIS signal is spatially transformed to obtain the best correspondence pixel by pixel with the UV signal. This transformation comprises translation, rotation and scaling. Then, the vapor absorption signal was calculated $\left(\ln \left(I_{0} / I\right)_{U V}-R \ln \left(I_{0} / I\right)_{V I S}\right)$.

At this point, the result is line-of-sight integrated. Thus, a deconvolution (inversion) algorithm is required to obtain the corresponding signal at the symmetry plane of the spray. This algorithm is applied to one half of the spray, thus the original absorption signal is divided into two halves (along spray axis), which are averaged before applying the deconvolution algorithm. The Onion-Peeling method is the most commonly used algorithm for numerical deconvolution (inversion) of a LoS attenuation signal [11, 12, 25, 26]. Nevertheless, in this work, the Three-Point Abel Inversion was chosen, as it has some advantages in terms of noise when comparing with the Onion-Pelling 27] method. Besides, it was combined with the Tikhonov regularization methodology [26, 28, to minimized 
the influence of noise over deconvoluted signal. A regularization parameter has to be optimized for each radial profile of the spray along its axis, to improve accuracy of the algorithm. In this regard, an automatic selection method was employed, proposed by Åkesson et al. [28].

Eventually, equation 2 is utilized to calculate the vapor fuel partial density $\left(\rho_{v f}\right)$ from the deconvoluted attenuation signal. It has to be noted that the form at which this equation has been presented corresponds to the calculation of the LoS averaged partial density $\left(\overline{\rho_{v f}}\right)$. When applying this equation to the symmetry plane, the optical path $(\mathrm{L})$ considered is the minimum spatial unit (i.e. 1 pixel). To solve the possible dependence of the absorption coefficient with local temperature, a mixing model (state relationship) was employed, which is based on the assumption that the mixture state corresponds to the result of an adiabatic mixing process. Therefore, it is possible to correlate the local fuel partial density with its temperature. A detailed description is presented in 29]. Pressure within the spray has been assumed to be the same as for the surrounding gas. The state relationship was also utilized to obtain the fuel mass fraction distribution from the fuel partial density.

\section{Results and discussion}

\subsection{Absorption coefficient}

As previously presented, absoprtion coefficient measurements were performed according to the conditions in table 2 . Figure 3 shows an assembled image of attenuation at $280 \mathrm{~nm}$, which was obtained by injecting $54.37 \mathrm{mg}$ of DEC (actual injection timing from -80.5 to $-45 \mathrm{CAD}$ ), at in-cylinder conditions corresponding to the LD case. The overall spatial distribution of attenuation is practically homogeneous along the whole combustion chamber, so that optics shifting was not necessary for calibration. Therefore, attenuation at 280 and $560 \mathrm{~nm}$ was measured only at the centre of the optical access. Images also indicate the existence of small scale inhomogeneities, which are most probably due to beam steering, as such a pattern can also be observed in the background part of schlieren 
images [21. For all cases, measured attenuation at $560 \mathrm{~nm}$ was one order of magnitude lower than the stardard deviation from the image sample, i.e. signal is in the range of the background noise, which confirmed the initial hypothesis of no absorption by vapor in the visible.

In figure 4, average $\varepsilon$ values at $280 \mathrm{~nm}$ are presented for the investigated fuels. The first point (lowest temperature) of each series correspond to the value obtained at $0.1 \mathrm{MPa}$ and $298 \mathrm{~K}$ (STP), measured with the spectrometer. The rest of the points correspond to different combinations of mean temperature and pressure inside the cylinder at the moment of image acquisition. The comparison of two series with similar in-cylinder pressure at TDC enables the analysis of the temperature influence, while the comparison of two series with similar incylinder temperature at TDC makes it possible to study the effect of pressure. For the sake of clarity, different engine conditions are only shown for HEPB3. For this fuel, the absorption coefficient corresponding to $560 \mathrm{~nm}$ has been also included. Results show that this value is negligible, confirming the hypothesis that no absorption occurs at this wavelength.

The absorption coefficient can be observed to increase with the fraction of aromatic fuels (HEPB1 to HEPB3), while DEC and 50DEC present significantly lower $\varepsilon$ values. For all the fuels, a large difference in $\varepsilon$ is observed between STP and engine conditions. Note that the STP is intended here to be used only as a reference for the in-cylinder measured values. Furthermore, little sensitivity to in-cylinder pressure and temperature can be observed for the different blends. This is consistent with results presented by Zhang et al. 11], who reported a large reduction of the absorption coefficient when pressure and temperature increased. However, above a certain level (ambient pressure above $3 \mathrm{MPa}$ and ambient temperature above $650 \mathrm{~K}$ ) this sensitivity tends to decrease. Moreover, the sensitivity is clearly dependent on the type of molecule, as they report variations of the absorption coefficient around $10 \%$ for 1,3-Dimethylnaphtalene and $60 \%$ for $\alpha$-Methylnaphtalene, when temperature changed from 575 to 650 $\mathrm{K}$ at $3 \mathrm{MPa}$ ambient pressure. Yamakawa et al. 12] also reported that the absorption coefficient of p-xylene is almost not affected by thermodynamic con- 
ditions above $1.5 \mathrm{MPa}$ and $400 \mathrm{~K}$. Summing up, literature results conclude that the sensitivity of $\varepsilon$ to ambient thermodynamic conditions tends to minimize or even disappear at high pressures and temperatures, which is consistent with the results presented in this work.

A similar behaviour is observed for DEC and 50DEC. Furthermore, for these two low absorption fuels two different energizing times have been used (table 2), and therefore two $\varepsilon$ values can be observed at each ambient condition, which fall onto each other. On the one hand, this indicates that the procedure is independent of the injected mass. On the other hand, it also confirms that the hypothesis of complete evaporation of the fuel is valid for the investigated conditions, and discards any systematic error on $\left(\overline{\rho_{v f}}\right)$ calculation due to spray wall impingement or liquid formation.

\subsection{Signal-to-noise considerations for spray measurements}

LoS attenuation along spray axis is depicted for HEPB3 (upper plot) and 50DEC (lower plot) in figure 5 for $280 \mathrm{~nm}, 560 \mathrm{~nm}$ and the corresponding difference. Data correspond to NO conditions.Closer to the nozzle, visible and UV signals are similar as scattering dominates due to the low amount of vaporized fuel. At some point (depending of the amount of vaporized fuel and the absorption coefficient), the visible signal becomes lower and the single contribution of the vapor absorption can be measured. In this figure, it is possible to see that the net vapor absorption signal calculated for 50DEC is of the same order of magnitude as the attenuation obtained for the visible wavelength. If this last signal is considered as noise (mainly caused by beam steering), the corresponding signal-to-noise ratio (calculated between 25 and $50 \mathrm{~mm}$ ) is 1.80 . In contrast, the attenuation of HEPB3 at $280 \mathrm{~nm}$ is in general one order of magnitude higher. Even closest to the nozzle, where the dense liquid region is located, some vapor absorption signal can be detected. In this case, the signal-to-noise ratio is 26.46. Regarding DEC, a similar calculation was performed resulting on a signal-to-noise ratio of 4.89 , which is closer to 50DEC than to HEPB3. These results evidence the advantage of usign highly absorbing fuels to obtaine 
reliable measurements under the investigated conditions.

\subsection{Spray measurements}

The $\varepsilon$ calibration procedure has to be validated to guarantee the reliability of results. For this purpose, the vapor fuel distribution was measured and compared for the three HEPB blends at LD conditions. In figure 6 (upper plot), the partial density of the three fuels are compared. A peak can be observed in the fuel concentration evolution, which is a good estimation of the location of the stabilized liquid length. Similar fuel concentrations were obtained for the three fuels downstream of the peak, where the spray is fully vaporized. This result is consistent with the fact that mass flow rate and spray momentum flux show almost no change among blends, which should result in a very similar mixing process for all three cases 21, 29. For distances shorter than the maximum liquid length, the fraction of each component in the vapor phase is unknown, and thus the absorption coefficient cannot be strictly applied, as it was obtained only for a fully vaporized mixture. For this reason, differences larger than expected are observable upstream of the peak values of each case.

The second aspect that needs to be validated is the sensitivity of $\varepsilon$ to incylinder pressure and temperature. According to the results presented in figure 4 a constant value of $\varepsilon$ has been used to obtain $Y_{f}$ for each fuel, under different thermodynamic conditions. The vapor fuel mass fraction of HEPB3 is shown in figure 6 (lower plot), for the three operating conditions described in figure 1. Data corresponds to the value along the spray axis. The X-coordinate of each curve has been normalized with the equivalent diameter [30, which is defined as $\phi_{e q}=d_{0} \sqrt{\rho_{f} / \rho_{a}}$, where $d_{0}$ is the nozzle exit diameter, $\rho_{f}$ is the fuel density and $\rho_{a}$ is the ambient gas density. The normalization process should enable the comparison of all three cases at the same entrainment coordinate. The three distributions are observed to collapse after the normalization, which confirms that results are consistent. Therefore, it can be stated that $\varepsilon$ is independent of thermodynamic conditions for the fuels and operating conditions considered in this study, as expected. 
In figure 7 , the vapor mass fraction on the spray axis is compared for the three ambient densities presented in figure 1 and DEC, 50DEC and HEPB3. In the highly dense liquid region (i.e. first $10-20 \mathrm{~mm}$ ), results for 50DEC are not plotted due to the extremely high noise observed. The low amount of vaporized fuel, in combination with a low absorption coefficient, leads to large uncertainties on the experimental data. Thus, despite offering promising results with DEC, the methodology described in this work can be observed not to be sensitive enough to characterize this region if low absorption fuels such as the 50DEC are considered. Nevertheless, it is important to highlight that near the maximum liquid length, the technique is able to measure the vapor fuel distribution in presence of liquid, even for 50DEC. Although uncertainties on the accuracy within this region exist due to the presence of droplets, this does not rule out the qualitative evaluation of the vaporized fuel mass fraction.

Downstream of the maximum vapor mass fraction, liquid is completely evaporated, air entrainment continues and fuel mass fraction decreases until the tip of the spray is reached. Along this region, mass fraction distribution for both HEPB3 and DEC coincide. However, it is not the case for 50DEC. For the NO and LD cases, higher values of $Y_{f}$ were obtained for this fuel in comparison with the other two. When 50DEC and HEPB3 are compared (from 25 to 35 $\mathrm{mm}$ ), differences are around $20 \%$ for the NO point and $40 \%$ for LD. As from the previous section, the calibration methodology was able to characterize low $\varepsilon$ values. However, it can be observed (figure 4 that all the fuels present a similar standard deviation, despite the fact that the value of $\varepsilon$ can be more than one order of magnitude different. The main consequence is that, while for HEPB3 the deviation accounts for a maximum of $5 \%$ of the mean value, in the case of 50DEC the standard deviation reaches almost $50 \%$ of the mean value. This leaves much uncertainty over the calculated average value of $\varepsilon$, which directly affects $Y_{f}$ distributions estimations. Based on these arguments on the calibration, as well as on the evolution of on-axis fuel mass fraction in figures 5 and 7 , it can be stated that 50DEC represents a limitation of sensitivity for 
the methodology described in this work. The minimum threshold in absorption coefficient for the adequate application seems to be between that of 50DEC and DEC, as the latter fuel seems to be enough to improve significantly accuracy and quality of results to acceptable levels.

Gas jet theory (e.g. [31]) shows that, in the fully vaporized region of the spray, the fuel mass fraction should have self-similar profiles. This means that fuel mass fraction normalized by the corresponding on-axis value $\left(\left(Y_{f} / Y_{f, c l}\right)\right.$ should only be dependent of the radial to axial coordinates $(R / X)$. From a similar point of view, the radii where $15 \%, 50 \%$ and $90 \%$ of $Y_{f, c l}$ is located should be a constant, if divided by the axial coordinate. This actually the type of result that is shown in Figure 8 for DEC, 50DEC and HEPB3 and the three test conditions defined in figure 1. Data below 15\% have not been considered in this analysis due to the large uncertainties observed in the outer regions of spray and the low signal-to-noise ratio, especially for the low absorption fuels.

The first thing to be noticed is that the data scatter is, in general, smaller for HEPB3 than for the other two fuels. Nevertheless, for 90\%, a certain variability is observed for all of them. It has been previously reported in the literature [26, 27. that the deconvolution algorithm introduces errors close to the axis. Besides, the numerical procedure followed in this work tends to flatten them around this region, hindering the accurate calculation of the radii. A second aspect to note is that, in general, radii values for the three fuels are similar. This suggests that the discrepancies for 50DEC, reported in figure 7, are related to the value of the absorption coefficient. As $\varepsilon$ has been shown to have no dependency on pressure or temperature, in practice it acts like a proportionality constant to convert attenuation into fuel concentration. Therefore, when profiles are normalized, the effect of the absorption coefficient is removed and the three fuels are similar. Finally, the flat trends observed for the normalized radii versus axial distance confirm that the radial profiles are self-similar in the fully vaporized region.

The normalization of radial profiles depends on the accuracy with which the numerical procedure is able to reconstruct the symmetry plane of the spray. As 
commented above, these algorithms tend to accumulate errors at the inner parts of the radial profiles [27]. To determine the effect of this issue on the global shape of the inverted profiles and its normalization, a fitted Gaussian curve has been compared with the experimental data, which is a profile shape usually found in the literature. The fitting algorithm is based on the maximum gradient descent methodology (according to Palomares [32]) and the experimental data considered for this purpose is the one comprised between $15 \%$ and $90 \%$ of the $Y_{f, c l}$. In figure 9 (upper plot), a comparison between experimental and fitted radial profiles is shown. Data corresponds to HEPB3 and DEC, at NO thermodynamic conditions. It can be seen that the agreement between experimental and fitted distributions is high in the range considered for the calculation. However, as expected, the fitted curve presents higher values near the spray axis. This comparison also reveals another region (especially for the DEC profiles), where the Gaussian trend is not followed, namely the edge of the spray. In figure 9 (lower plot), the ensemble averaged normalized profiles calculated between 25 and $35 \mathrm{~mm}$ for HEPB3 and DEC at NO conditions are shown. In this case, it is possible to see that the fitted profiles of the two fuels are more similar than the experimental ones, which highlights the effect of noise over the deconvolution algorithm. In case of DEC, with relatively higher noise, the experimental profile does not even present a Gaussian shape, which is a more accurate description for HPB3 measurements.

\section{Conclusions}

The UV-VIS LAS technique has been proposed to characterize the air-fuel mixing process of two low absorption fuels (i.e. DEC and 50DEC). Three additional fuels with progressively higher absorptivity (HEPB1, HEPB2 and HEPB3) have also been characterized in order to compare and evaluate the accuracy and reliability of the technique and the results obtained for the first ones.

A calibration procedure has been designed to obtain in-situ measurements 
of the absorption coefficient, using the same optical set-up as the one proposed for spray measurements. For the conditions and fuels used in the calibration procedure, the following conclusions were obtained:

- Fuel-air mixture inside the chamber was found to be homogeneous and the absorption coefficient calculation was found to be independent of fuel concentration

- Experimental results show that the methodology is sensitive to fuel properties.

- Measured $\varepsilon$ values suggested a negligible sensitivity of this parameter to pressure or temperature. These results have been also validated experimentally, thanks to the consistence observed between fuel distributions measured at different engine operating conditions

- It has been possible to characterize $\varepsilon$ for low absorption fuels like DEC and 50DEC. However, results present uncertainties, which could even achieve the $50 \%$ of the average value.

The values of $\varepsilon$ have been used to obtain the fuel vapor distribution for DEC, 50DEC and HEPB3, from which following conclusions have been drawn:

\section{- Measurements of the vapor fuel distribution near liquid length} have been obtained for all the fuels, although uncertainties exist in regions where droplets are present.

- Accuracy and quality of results decrease with the absorption coefficient. Similar results have been obtained for HEPB3 and DEC, while for 50DEC values higher than expected have been measured.

Considering all the foregoing arguments, the methodology described in this paper is of limited applicability when trying to characterize fuels with absorption properties in the range of 50DEC. Fuels with $\varepsilon>11 \mathrm{lmol}^{-1} \mathrm{~cm}^{-1}$, such as DEC, are suitable for this methodology. Furthermore, the larger the $\varepsilon$ values, 
the higher the validity of the results, as signal strength improves with this parameter.

\section{Acknowledgements}

This work was partially funded by the Spanish Ministry of Science and Technology through project TRA2011-26359. In addition, the authors acknowledge that some equipment used in this work has been partially supported by FEDER project funds (FEDER-ICTS-2012-06), framed in the operational program of unique scientific and technical infrastructure of the Ministry of Science and Innovation of Spain.

\section{References}

[1] D. L. Siebers, B. S. Higgins, Flame lift-off on direct-injection diesel sprays under quiescent conditions, SAE Technical Paper 2001-01-0530.

[2] H. Zhao, Laser Diagnostics and Optical Measurement Techniques in Internal Combustion Engines, SAE International Warrendale, 2012.

[3] C. A. Idicheria, L. M. Pickett, Quantitative mixing measurements in a vaporizing diesel spray by rayleigh imaging, SAE Technical Papers 200701-0647.

[4] L. M. Pickett, J. Manin, C. L. Genzale, D. L. Siebers, M. P. Musculus, C. A. Idicheria, Relationship between diesel fuel spray vapor penetration/dispersion and local fuel mixture fraction, SAE International Journal of Engines 4 (1) (2011) 764-799.

[5] J. M. Desantes, J. V. Pastor, J. M. Pastor, J. E. Juliá, Limitations on the use of the planar laser induced exciplex fluorescence technique in diesel sprays, Fuel 84 (18) (2005) $2301-2315$. 
[6] C. Schulz, V. Sick, Tracer-lif diagnostics: Quantitative measurement of fuel concentration, temperature and fuel/air ratio in practical combustion systems, Progress in Energy and Combustion Science 31 (1) (2005) 75-121.

[7] T. Fang, R. E. Coverdill, C. F. Lee, R. A. White, Air-fuel mixing and combustion in a small-bore direct injection optically accessible diesel engine using a retarded single injection strategy, Fuel 88 (11) (2009) 2074 - 2082.

[8] W. Zeng, M. Xu, G. Zhang, Y. Zhang, D. J. Cleary, Atomization and vaporization for flash-boiling multi-hole sprays with alcohol fuels, Fuel 95 (0) (2012) $287-297$.

q [9] E. Mancaruso, B. M. Vaglieco, Spectroscopic measurements of premixed combustion in diesel engine, Fuel 90 (2) (2011) 511 - 520. doi:http://dx.doi.org/10.1016/j.fuel.2010.09.052

URL http://www.sciencedirect.com/science/article/pii/ S0016236110005247

[10] M. Suzuki, K. Nishida, H. Hiroyasu, Simultaneous concentration measurement of vapor and liquid in an evaporating diesel spray, SAE Technical Paper 930863.

[11] Y.-Y. Zhang, T. Yoshizaki, K. Nishida, Imaging of droplets and vapor distributions in a diesel fuel spray by means of a laser absorption-scattering technique, Applied Optics 39 (33) (2000) 6221-6229.

[12] M. Yamakawa, D. Takaki, T. Li, Y.-Y. Zhang, K. Nishida, Quantitative measurement of liquid and vapor phase concentration distribution in a d.i. gasoline spray by the laser absortion scattering (LAS) technique, SAE Technical Paper 2002-01-1644.

[13] Y.-Y. Zhang, K. Nishida, T. Yoshizaki, Characterization of droplets and vapor concetration distributions in split-injection diesel sprays by processing UV and visible images, JSME International Journal, Series B: Fluids and Thermal Engineering 46 (2003) 100-108. 
[14] Y.-Y. Zhang, K. Nishida, Vapor distribution measurement of higher and lower volatile components in an evaporating fuel spray via laser absorption scattering (LAS) technique, Combustion Science and Technology 179 (5) (2007) 863-881.

[15] M. Chato, S. Fukuda, K. Sato, T. Fujikawa, R. Chen, Z. Li et al., Fuel spray evaporation and mixture formation processes of ethanol/gasoline blend injected by hole-type nozzle for disi engine, SAE International Journal of Engines (2012) 1836-1846.

[16] V. Bermúdez, J. M. García, M. S. Juliá, J. E., Engine with optically accessible cylinder head: A research tool for injection and combustion processes, SAE Technical Papers 2003-01-1110.

[17] F. Payri, J. V. Pastor, J.-G. Nerva, J. M. Garcia-Oliver, Lift-off length and KL extinction measurements of biodiesel and fischer-tropsch fuels under quasi-steady diesel engine conditions, SAE International Journal of Engines 4 (2) (2011) 2278-2297.

[18] T. P. Billings, J. A. Drallmeier, A detailed assessment of the infrared extinction technique for hydrocarbon vapor measurements in a controlled two-phase flow, Atomization and Sprays 4 (1) (1994) 99-121.

[19] A. Kokhanovsky, E. Zege, Optical properties of aerosol particles: A review of approximate analytical solutions, Journal of Aerosol Science 28 (1) (1997) 1-21.

URL http://www.scopus.com/inward/record. url?eid=2-s2.0-0031060450\&partnerID=40\&md5= ff2882fb1416ecd34541853a1664ec7c

[20] H. Zhang, Approximate calculation of extinction coefficient, Journal of Physics D: Applied Physics 23 (12) (1990) 1735-1737. doi:10.1088/0022-3727/23/12/038. URL http://www.scopus.com/inward/record. 
url?eid=2-s2.0-0025560753\&partner ID=40\&md5=

39e7fd8953e8ca03b3d06112df9f7127

[21] J. V. Pastor, J. M. García-Oliver, V. Bermúdez, C. Micó, Spray characterization for pure fuel and binary blends under non-reacting conditions, SAE Technical Papers 2014-01-1407.

[22] J. Gao, K. Nishida, Laser absorption-scattering technique applied to asymmetric evaporating fuel sprays for simultaneous measurement ofvapor/liquid mass distributions, Applied Physics B: Lasers and Optics (2010) $1-11$.

[23] W. Bosch, The fuel rate indicator: a new instrument for display of the characteristic of individual injector, SAE Technical Paper 660749.

[24] R. Payri, F. J. Salvador, J. Gimeno, G. Bracho, A new methodology for correcting the signal cumulative phenomenon on injection rate measurements, Experimental Techniques 32 (1) (2008) 46-49.

[25] K. Nishida, J. Gao, T. Manabe, Y. Zhang, Spray and mixture properties of evaporating fuel spray injected by hole-type direct injection diesel injector, International Journal of Engine Research 9 (2008) 347-360.

[26] K. J. Daun, K. A. Thomson, F. Liu, G. J. Smallwood, Deconvolution of axisymmetric flame properties using Tikhonov regularization, Applied Optics 45 (2006) 4638-4646.

[27] C. J. Dasch, One-dimensional tomography: a comparison of Abel, oniopeeling, and filtered backprojection methods, Applied Optics 31 (1992) 1146-1152.

[28] E. O. Åkesson, K. J. Daun, Parameter selection methods for axisymmetric flame tomography through Tikhonov regularizaron, Applied Optics 47 (3) (2008) 407-416. 
[29] J. V. Pastor, J. M. García-Oliver, J. M. Pastor, W. Vera-Tudela, Onedimensonal diesel spray modelling of multi-component fuels, Atomization and Sprays 25 (6) (2014) pages 485-517.

[30] M. W. Thring, M. P. Newby, Combustion length of enclosed turbulent jet flames, Symposium (International) on Combustion 4 (1) (1953) $789-796$.

[31] D. B. Spalding, Combustion and mass transfer, no. ISBN 0-08-022105-8, 1979.

[32] A. Palomares, Estudio del proceso de inyección diesel mediante visualización y procesado de imágenes, Universitat Politècnica de València, 2001. 


\begin{tabular}{cccccc}
\hline Component & DEC & 50DEC & HEPB1 & HEPB2 & HEPB3 \\
\hline n-heptane & 0 & 0 & 95.3 & 93.5 & 91.7 \\
n-decane & 100 & 50 & 0 & 0 & 0 \\
n-dodecane & 0 & 0 & 0.5 & 0.6 & 0.8 \\
n-hexadecane & 0 & 50 & 1.4 & 1.9 & 2.4 \\
n-octadecane & 0 & 0 & 0.9 & 1.3 & 1.7 \\
n-eicosane & 0 & 0 & 0.6 & 0.8 & 1.1 \\
1-methylnaphtalene & 0 & 0 & 1.2 & 1.7 & 2.1 \\
n-butylbenzene & 0 & 0 & 0.1 & 0.2 & 0.2 \\
\hline
\end{tabular}

Table 1: Fuel composition in percentage (mass) for the present study. 


\begin{tabular}{|c|c|c|c|c|c|c|}
\hline Fuel & $\begin{array}{c}\rho_{\mathrm{f}} \text { at } 373 \mathrm{~K} \\
{\left[\mathrm{~kg} / \mathrm{m}^{3}\right]}\end{array}$ & Test point & $\begin{array}{c}P_{\text {inj }} \\
{[\mathrm{MPa}]}\end{array}$ & $\begin{array}{c}\text { Energizing } \\
\text { Time }[\mu \mathrm{s}]\end{array}$ & $\begin{array}{l}\text { Total injected } \\
\text { mass }[\mathrm{mg} / \mathrm{cc}]\end{array}$ & $\underset{\text { of interest }}{\text { CAD }}$ \\
\hline DEC & 669.1 & $\begin{array}{l}\mathrm{LD}, \mathrm{NO}, \\
\mathrm{HT}\end{array}$ & 150 & 4500,9000 & $37.53,54.37$ & $\begin{array}{c}0,6 \\
12,18\end{array}$ \\
\hline $50 \mathrm{DEC}$ & 693.9 & $\begin{array}{l}\mathrm{LD}, \mathrm{NO}, \\
\mathrm{HT}\end{array}$ & 150 & 4500,9000 & $39.70,64.03$ & $\begin{array}{c}0,6 \\
12,18\end{array}$ \\
\hline HEPB3 & 668.3 & $\begin{array}{l}\mathrm{LD}, \mathrm{NO}, \\
\mathrm{HT}\end{array}$ & 150 & 9000 & 60.25 & $\begin{array}{c}0,6,12, \\
18,24,30\end{array}$ \\
\hline HEPB2 & 666.3 & $\mathrm{LD}$ & 150 & 9000 & 60.07 & $\begin{array}{c}0,6,12, \\
18,24,30\end{array}$ \\
\hline HEPB1 & 664.2 & $\mathrm{LD}$ & 150 & 9000 & 59.88 & $\begin{array}{c}0,6,12, \\
18,24,30\end{array}$ \\
\hline
\end{tabular}

Table 2: Test conditions and fuel properties for the absorption coefficient calibration. 

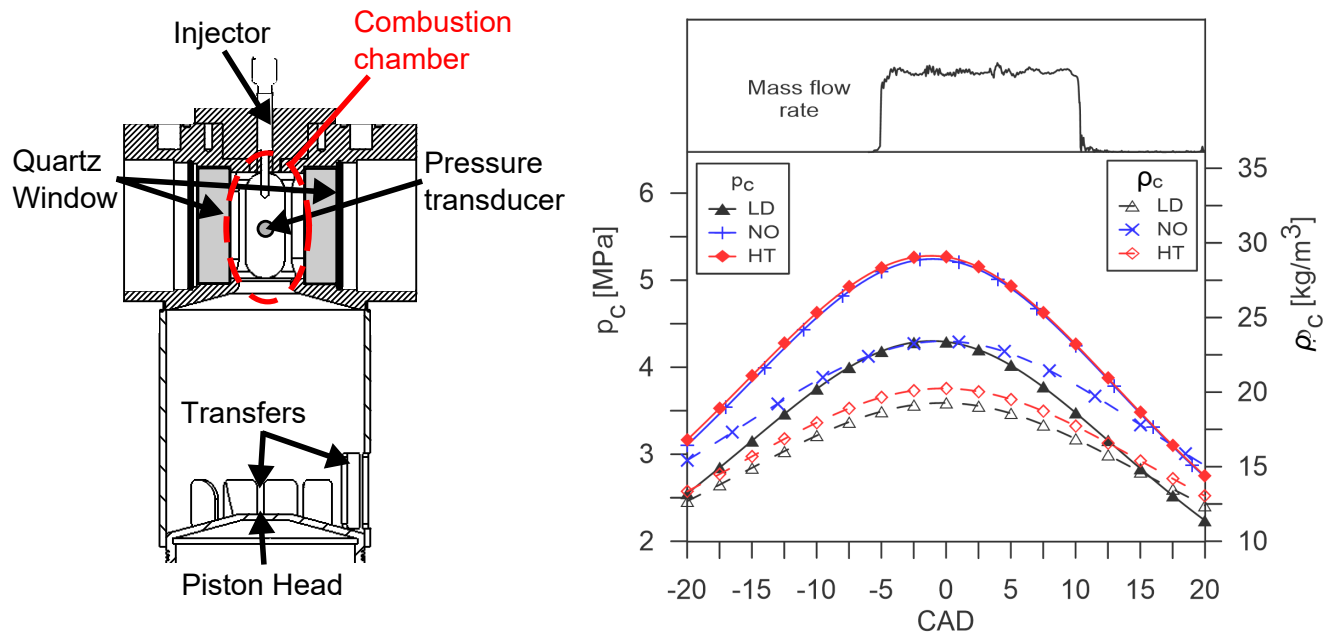

Figure 1: Scheme of the arrangement of the cylinder head and the liner (left). Evolution of in-cylinder pressure and density for the three operating points (right). 


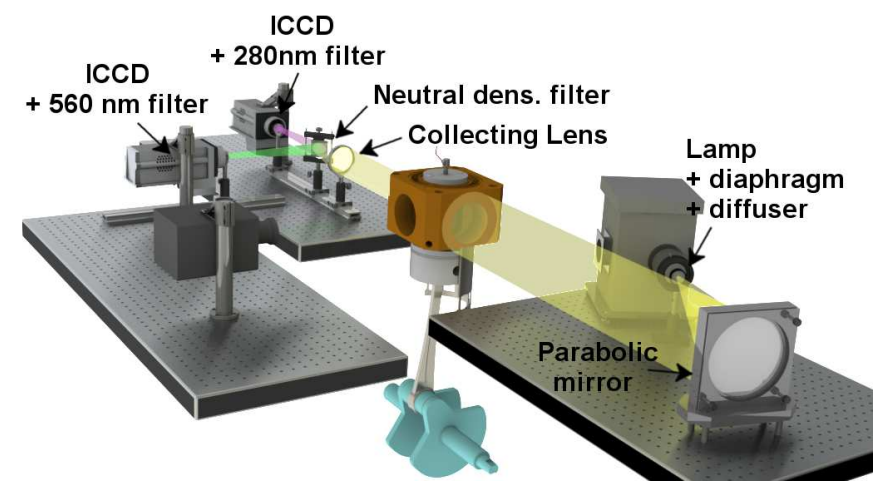

Figure 2: Scheme of the UV-VIS LAS optical set-up. 


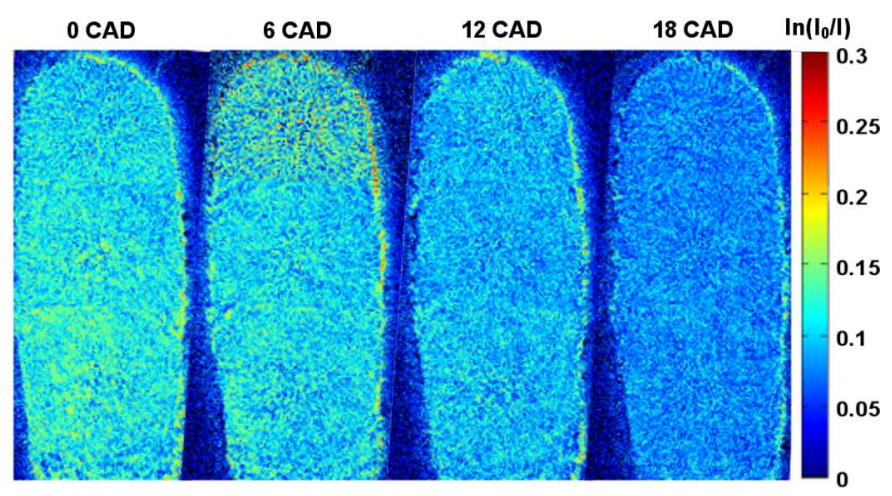

Figure 3: Example of in-cylinder homogeneous attenuation corresponding to $280 \mathrm{~nm}$ for DEC, at LD thermodynamic conditions. 


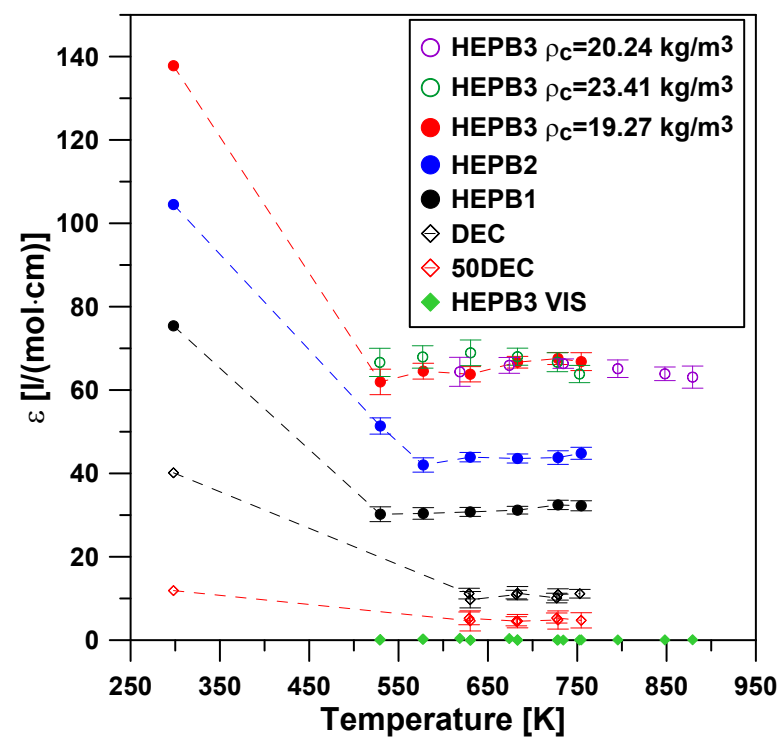

Figure 4: UV Absorption coefficient as a function of temperature for all the fuels and different engine conditions. VIS absorption coefficient is also included for HEPB3 

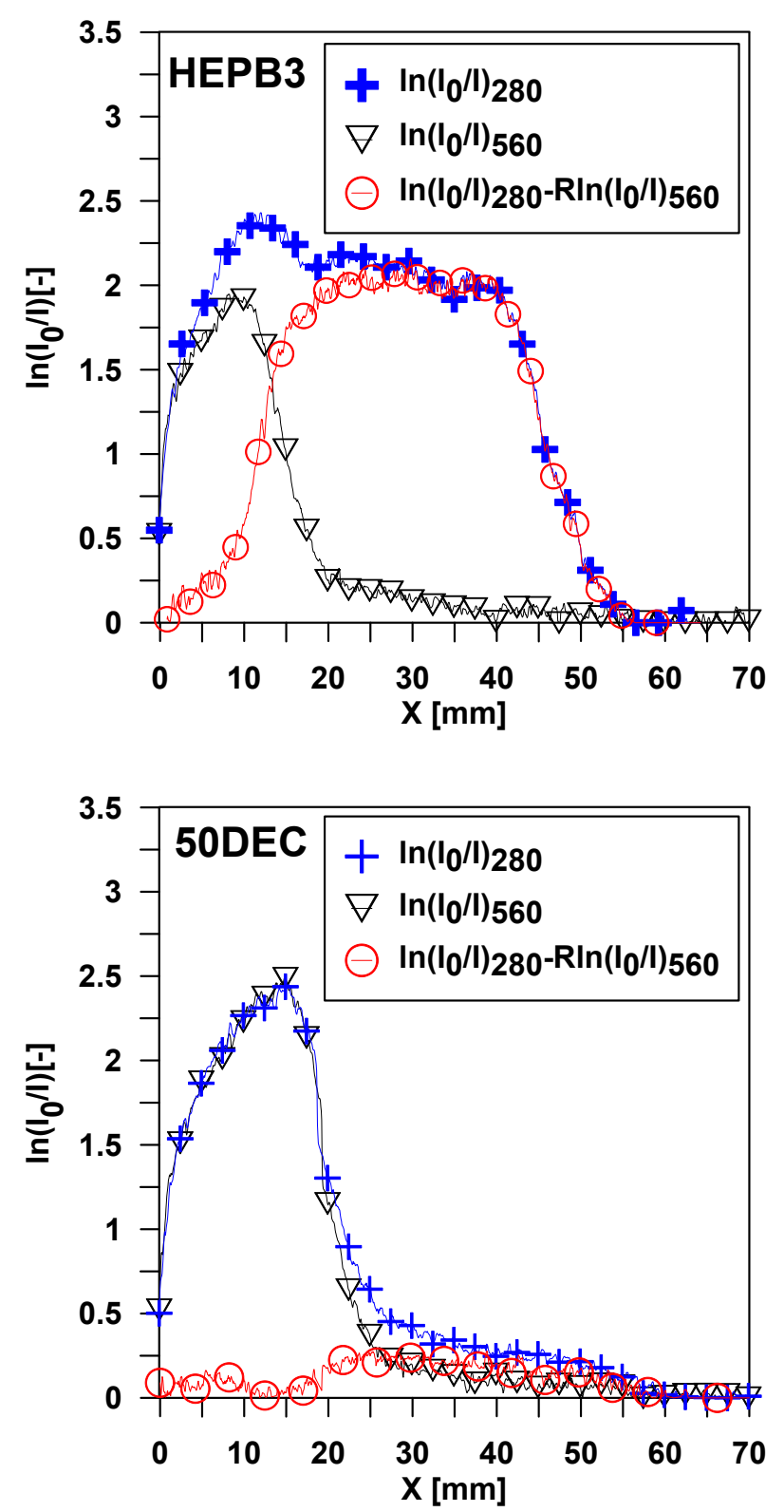

Figure 5: LoS attenuation for 560 and $280 \mathrm{~nm}$ on the spray axis for HEPB3 (upper plot) and 50DEC (lower plot). Data corresponds to NO conditions at -3 CAD before TDC. 

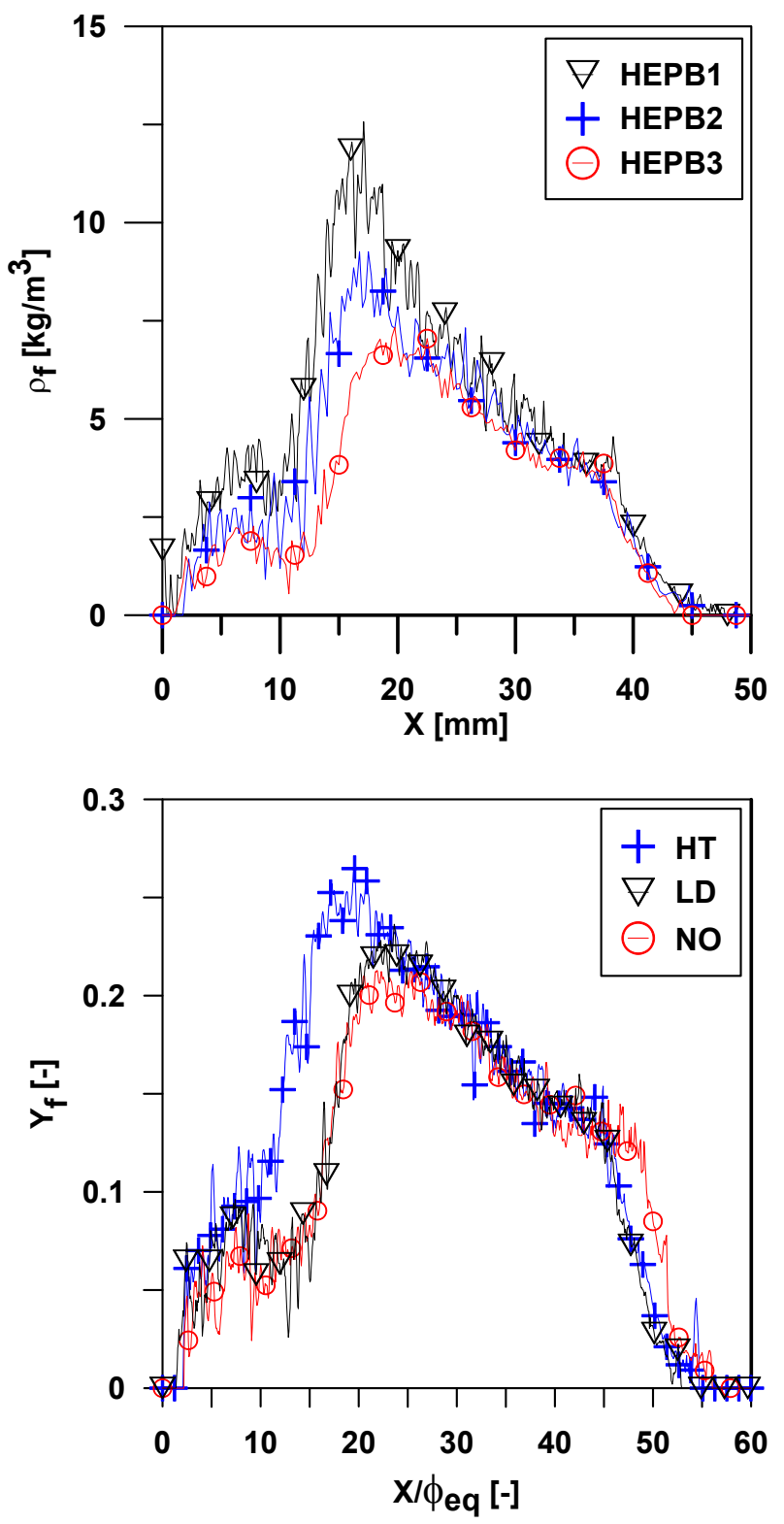

Figure 6: On-axis distribution of $\rho_{v f}$ for the three mixtures of HEP and HAF, at LD conditions (upper plot).On-axis $Y_{f}$ distribution of HEPB3, for the three conditions defined in figure 1 (lower plot). All the data were obtained at -3 CAD before TDC. 

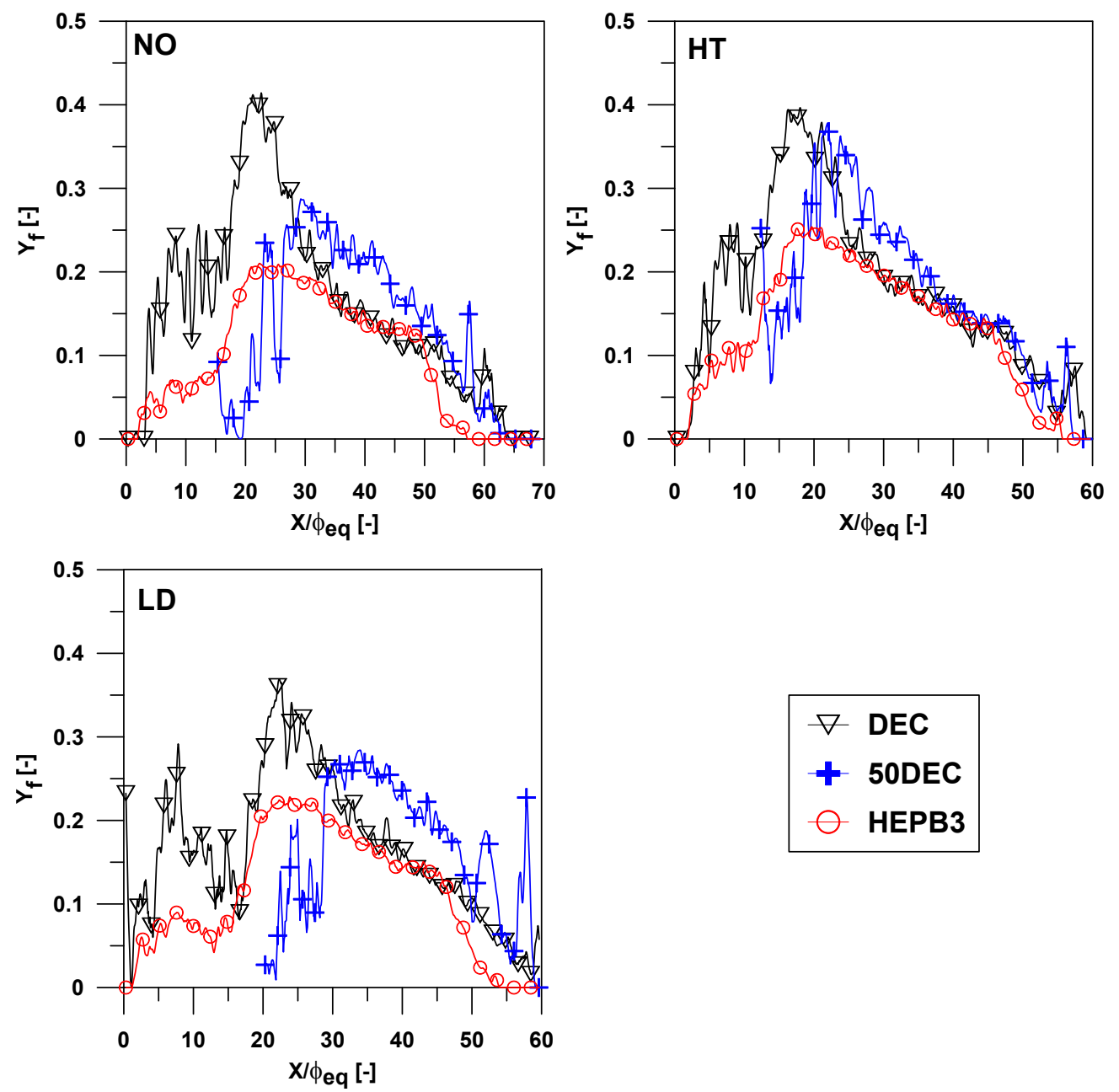

Figure 7: On axis distribution of $Y_{f}$ of DEC, 50DEC and HEPB3. Data corresponds to the thermodynamic conditions defined in figure 1 at -3 CAD before TDC. 


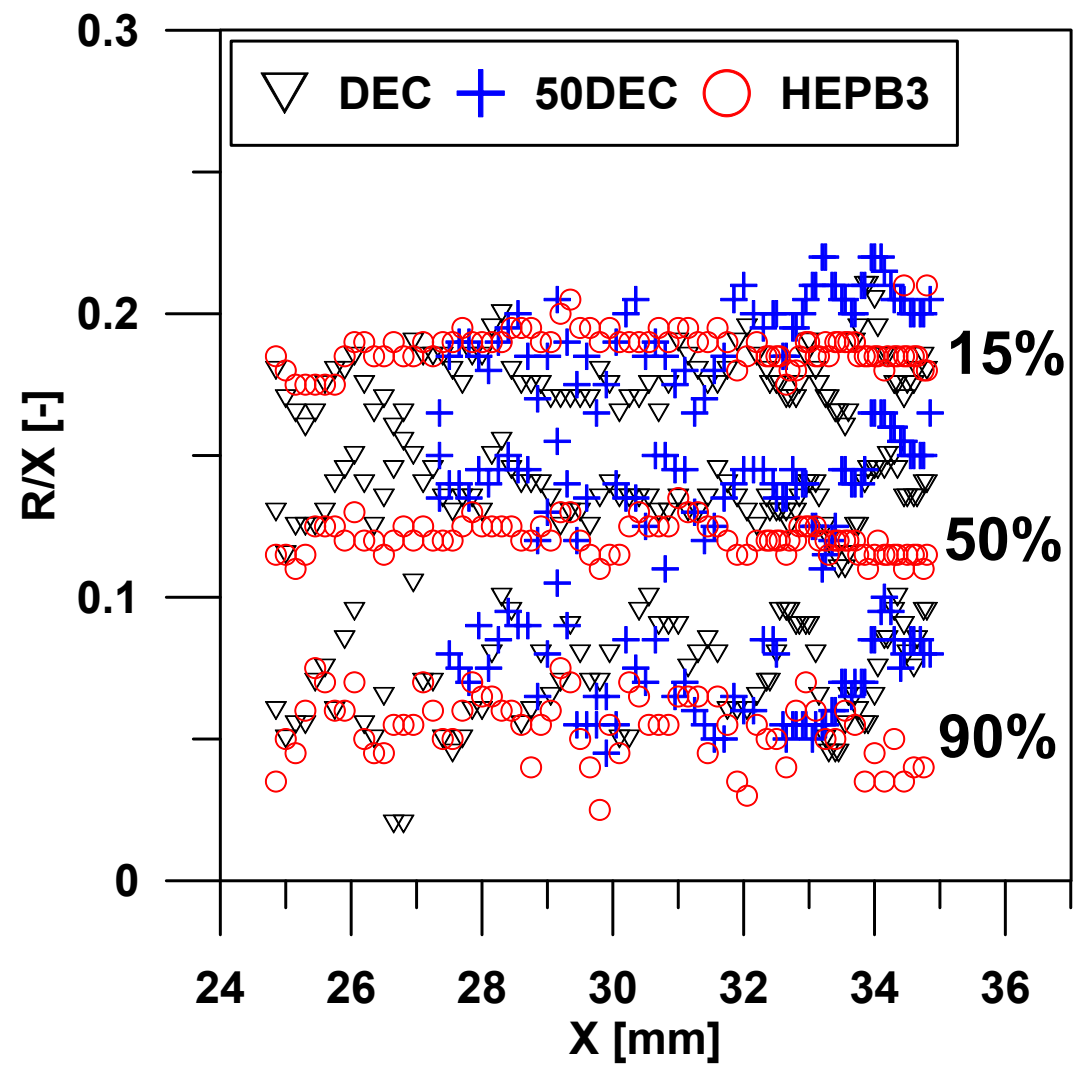

Figure 8: Radii for $15 \%, 50 \%$ and $90 \%$ of $Y_{f, c l}$ fir DEC, 50DEC and HEPB3 along the spray axis. Data corresponds to the three operating conditions defined in figure 1 at -3 CAD before TDC. 

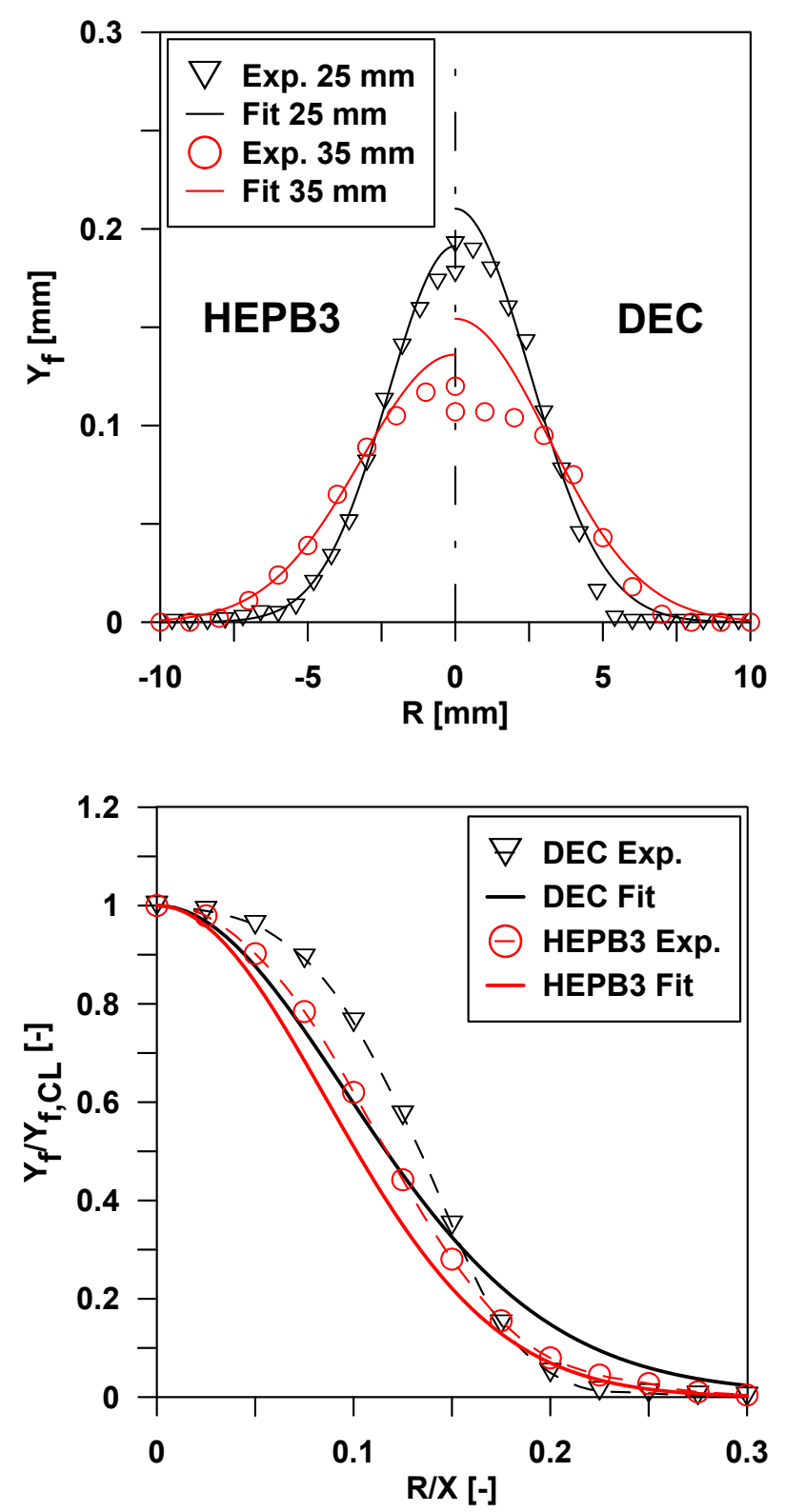

Figure 9: Comparison between experimental and fitted exponential curve for DEC and HEPB3, at 25 and $35 \mathrm{~mm}$ (upper plot). Comparison between experimental and fitted normalized profiles, averaged between 25 and $35 \mathrm{~mm}$ (lower plot). Data corresponds to NO thermodynamic conditions at -3 CAD before TDC. 